\title{
Study of the Construction of a System to Investigate Actual Parking Conditions using a Smartphone
}

\author{
Youngwoo Lee \\ Dept. of Civil Engineering, Daegu University \\ 201, Daegudae-ro, Gyeongsan-si, Gyeongsangbuk-do, 38453 Republic of Korea \\ lyw209@daegu.ac.kr
}

\begin{abstract}
The demand for parking surveys has increased significantly. Despite the increased. demand for parking surveys, however, there has been little research and technological developments. Existing parking surveys are carried out by inspectors recording the vehicle license numbers on paper and showing their approximate location on map. This method is time consuming and it is difficult to ensure the accuracy of the location data. In addition, it has the problem of the additional cost and time to computerize the survey data. Therefore, this study evaluated a parking survey method utilizing a smartphone to overcome the limitations of existing parking survey. First, the essential items in a parking survey were identified and defined. Based on this, a smartphone application was developed for parking surveys. Using the developed application, a system was constructed to collect the parking survey data, and the necessary software to analyze and express the collected data was developed. If the result of this studyean be applied to various parking surveys, then it is expected to be effeetive in reducing the social costs through more efficient parking surveys and prevent unnecessary duplicated surveys by the efficient management of parking survey data. Furthermore, through the tabulation and visualization of data through maps, it is expected to help understand more comprehensively and three-dimensionaly the actual parking situation in the targeted survey area.
\end{abstract}

Keywords: Parking Survey, Smartphone Application, Parking Survey Item, GPS, Wireless Mobile Communication

\section{Introduction}

Parking in cities is becoming an increasingly serious traffic problem that is considered very difficult to solve. Despite the promotion of a variety of parking policies, there are limits to a fundamental resolution of the problem. Parking problems arise due to the need for destination parking in areas, such as the CBD and commercial zones, and a lack of garages in esidential areas. Lately, with regards to destination parking, the standards for the installation of attached parking lots are being strengthened legally and institutionally. Tenncrease the utilization of buildings, there are cases where the building owner voluntarily expands the number of parking spaces, but still the garage parking situation in residential areas is insufficient at night. In particular, for housing, such as apartments and multiplex houses, there are parking spaces but parking lot shortages are reaching serious levels in areas with detached houses.

Parking lot shortages lead to illegal parking and can be a major cause of traffic congestion and traffic accidents. In addition, parking problems in residential areas creates a situation that leads to social problems, such as dissent between people caused by these parking problems.

To alleviate the parking problems that are becoming increasingly serious, despite the range of policies, such as increasing the number of parking lots and the promotion of 
parking demand management, there are no effective solutions. The problem of the lack of garages in existing residential areas cannot be eased with parking demand management policies. Countermeasures that limit car ownership or expand the number of parking spaces are needed.

A policy that limits car ownership is, in reality, very difficult to implement when considering the various economic and social conditions. Parking lot supply policy should first ensure there is the required space for constructing a car park. On the other hand, securing land for the construction of parking lots is very difficult due to the recent highdensity urban development. Even if land is obtained, the increasing construction costs caused by rising land prices makes implementing a parking supply policy difficult.

The implementation of a reasonable and effective parking policy requires accurate analysis of parking problems associated with a variety of causes. This requires sufficient survey data on the basic actual parking conditions.

Therefore, in the Republic of Korea, through parking ordinances, mayors, county governors, or district heads decide on a survey area by comprehensively considering the administrative divisions, and zoning areas or regions, and conduct a survey of the supply and demand for parking lots every three years. This serves as the basis for the installation and management of parking lots.

In addition, survey results regarding actual parking conditions need to be submitted in the promotion of various development projects or in the permit process, so the demand for parking surveys has increased significantly. On the other hand, compared to the increase in demand for parking surveys, research and technological deveropment is at a critically low level.

Existing parking surveys are carried out by inspectors recording the vehicle license number on paper and showing the appreximate location on a map. This method takes too much time and it is difficult to ensure the accuracy of the location data. In addition, there is the problem of the additional costand time it takes to computerize the survey data.

Parking surveys are carried out for a large number of parked vehicles in a wide area. Therefore, the method that inspectors record information on paper is unsuitable for parking surveys due to lack of mobility Moreover, the reliability and accuracy of the data is reduced because the survey data is not computerized in real-time.

Some attempts bave been made to converge the latest state-of-the art IT technology in parking surveys to solve these problems.

Ha et al. (2014) provided an example of where a patent was registered for the development of a license prate recognition system using a mobile application. Lee and Kwon (2014) also conducted research to develop and utilize an automatic parking system that can carry out parkng surveys by mounting a camera on a vehicle to automatically recognize the license plates of parked cars. Despite this, existing research results have some limitations and do not satisfy all of the requirements for a parking survey.

This study evaluated a method to utilize smartphones to address the problems of parking surveys, which were carried out using the previous method. First, this study was condacted to identify and define the essential items in a parking survey. Based on this, a martphone application was developed for parking surveys. Using the developed application, a system was built to collect parking survey data, and the necessary software to analyze and express the collected data was developed.

\section{Related Research}

Most prior research on parking focused on parking demand forecasts, parking unit calculations or the development of parking policy. To conduct parking related research, basic data collection is needed and prior research has described the methodology used for parking surveys. Nevertheless, there have been few studies on parking surveys. 
Referring to previous research related to parking demand forecasts, Park et al. (1999), Park (2003), and Kwon et al. (2014) carried out research to develop a parking demand forecasting model by applying variables that affected the parking demand for apartment housing. Ahan and Lee (2010) conducted research to build a parking demand forecasting model for factory facilities in an industrial complex through a site survey. Lee (2008) carried out research on the methods to estimate the parking demand.

Referring to previous studies concerning parking units, Oh et al. (2006) conducted research to calculate the parking units using regression analysis after separating the data based on the city size and land use. Lee (2008) proposed parking unit application standards that can estimate the parking demand according to the facility type to compensate for the problems with parking unit calculations using existing site survey methods. Kwon et al. (2014) also developed a model to estimate parking units.

Lee et al. (2014) analyzed the parking situation with regards to parking turnover for off-street parking. Lee et al. (2013) carried out research on effective parking management by examining the plates of parked cars using a camera. With regards to research about onstreet parking, Hwang et al. (2010) analyzed the actual situation for streetparking on main roads and side streets, and Lee (2012) conducted research on illegal parking using an automated parking survey.

The most common survey methods used by researchers in previous studies, were surveys or questionnaires and site surveys. Survey on the actual parking conditions were conducted using CCTV or cameras directed at parked cars in limited spaces, such as offstreet parking.

Previous studies with a research objective of survey methods for actual parking conditions include Lee (2014), who conducted research to develop and utilize an automated parking system, but it was insufficient. In particular, there has been no prior research on parking surveys that incorporated smartphones. Therefore, in the present study, a parking survey was set up using the smartphone as a tool for parking surveys.

\section{Smartphone Application Development for Parking Surveys}

\subsection{Setting up Parking Survey Items}

To develop a smartphone application for parking surveys, the parking survey items must first be aesigned and developed. In this study, the items needed for parking surveys were set up through an analys is of previous studies and methods used in the surveys of supply of and demand for parking lots.

3.1.1 Inspector's Information: The input entry of inspector's information was minimized for personal information protection. This was set up to only enter the inspector's name to distinguish between inspectors so monitoring personnel can manage parking survey inspectors and make it possible for task transfer, such as where further investigations are required The system was developed to make it possible to input the survey inspector's name in Korean and English.

3.1.2 Survey Area Setting: Parking surveys can be carried out by categorizing according to the area or street depending on the reason for or specifications of the survey. In addition, because of the characteristics of the parking survey, which require an investigation of a wide area, an important factor in the success or failure of a parking survey is the inspector's precise knowledge of their own survey areas. Therefore, in this study, after verifying in advance the exact scope of their investigation, the inspectors can conduct the survey. To help the monitoring personnel determine if the parking inspector is doing the designated survey, the input item was set up to allow the scope of the survey to be specified by area or by street. 
3.1.3 Vehicle Classification: To perform parking surveys, vehicle classification of parked cars is vital. Even in 'Daegu City Surveys of Supply of and Demand for Parking Lots', vehicles are classified into three categories - passenger cars, small buses and trucks, and big buses and trucks. The category of passenger cars includes, vehicles such as general passenger cars, SUVs, passenger-type trucks, and vans with a seating capacity of less than sixteen passengers. Small buses and trucks include small trucks designed for the purpose of cargo, which are 1 to 2.5 tons, and buses with a seating capacity of 16-25 passengers. Large buses and trucks include large buses with a seating capacity of more than 25 passengers and heavy duty trucks and special vehicles, such as heavy-duty construction vehicles, which carry more than 2.5 tons of cargo.

In this study, vehicle classification also used the same classifications as the 'Daegu City Surveys of Supply of and Demand for Parking Lots', and was developed as a method that allows users to click on the menu for fast input. In addition, to minimize the inspector's input errors, every classified vehicle was numbered simultaneously and the selection was displayed in gray.

3.1.4 Separation of Legal and Illegal Parking: Parking surveys should be conducted by separating vehicles into legally and illegally parked-vehicles as needed in particular, the classification of legally and illegally parked vehicles is a very important factor in matters, such as parking spaces, parking demand management, and analysis of on-street parking.

Some examples of legal parking include where the vehicle is parked within the marked parking space, or more than half of the wehicle is parked within the lines, and where the vehicle is parked in an open area without preventing other traffic from passing. If the vehicle does not correspond to the definition of egal parking, it can be viewed as illegal parking. Some types of illegal parking are parking in the shoulder lane with a yellow solid line or dashed line, parking on a road with a enterline with no shoulder line, parking on a road with no marked parking spaces, and parking in places, such as on the open space in front of the building, a setback area, and a sidewalk.

Automatic parking sorveys that use items, such as cameras, have drawbacks; there is a high incidence of errors as the determination of legal and illegal parking is ambiguous. Moreover, the iniage can be analyzed to correct this but this takes a lot of time. In this study, while the inspectors are doing the parking survey, using an input method, which distinguishes between legal and illegal parking at the scene, the problem caused by unnecessary correction is minimized. The input method applied was to click on the smartphone screen and fill in a numeral according to the type of parking to prevent errors in the gray entry column.

3.1.5 Input of Vehicle License Plates: The license plates of vehicle in the Republic of Korea use both the Korean alphabet and numbers to make an identification code of seven to nine digits. For research on actual parking conditions and analysis, a survey on the vehicle license plate that identifies a parked vehicle is essential. In this study, a means for inspectors to enter the vehicle license plate information directly was developed. This feature is expected to be utilized in an analysis of parking characteristics, such as areaspecific classification and analysis of parked vehicles.

3.1.6 Location Information of Parked Vehicles: Location information of the parked vehicle is an essential element in the surveyed area or in the spatial analysis of a vehicle parked on the street. Therefore, in this study, the survey items were set up to enable the input of the location of parked vehicles according to latitude and longitude. The location information of the parked vehicles use GPS on the smartphones so the co-ordinates can be collected in real time. 
Compared to traditional methods of collecting location information of parked vehicles manually on paper, this method has the effect of significantly reducing the time and cost of on-site surveys and internal processing.

In addition, in the case where the survey method used a vehicle-mounted camera, this inherently caused large errors to occur because the location was based not on the precise location of the parked vehicle but on the location of the vehicle with the camera. On the other hand, the method developed in this study has a very strong advantage of accurate location information because it uses a smartphone adjacent to the parked vehicle making it possible to obtain the coordinate information. The input method used a system of clicking on the GPS confirmation button. Moreover, to confirm the location information, it was developed to show separately the latitude and longitude coordinates of the WGS84, which are the GPS coordinates.

\subsection{Results of Application Development}

When conducting parking surveys, items essential for the analysis include the inspector, survey site, and the identification and location information of parked vehicle. In this study, these essential items were subdivided and set up into eight specific items. In this study, by applying the set up parking survey items an Android-based smartphone application was developed for parking surveys. The screen was designed considering power loss so black was selected as the backgroun color.



Figure 1. Smartphone Application Developed for the Parking Surveys 
Table 1. Smartphone Application Developed for Parking Surveys






\section{Construction of a Parking Survey System using a Smartphone Application}

\subsection{Data Collection and Management System}

Parking surveys need to cover many vehicles over a very wide range; hence, even onsite surveys on actual parking conditions require considerable time and money. On the other hand, processing extensive field survey data including modifications also takes significant time and cost. Figure 2 shows the development of a system that can input and manage real-time survey data using a smartphone application that can investigate actual parking conditions on site.

\section{ANDROID KSOAP ASP SEFIJJEE PHIGE}
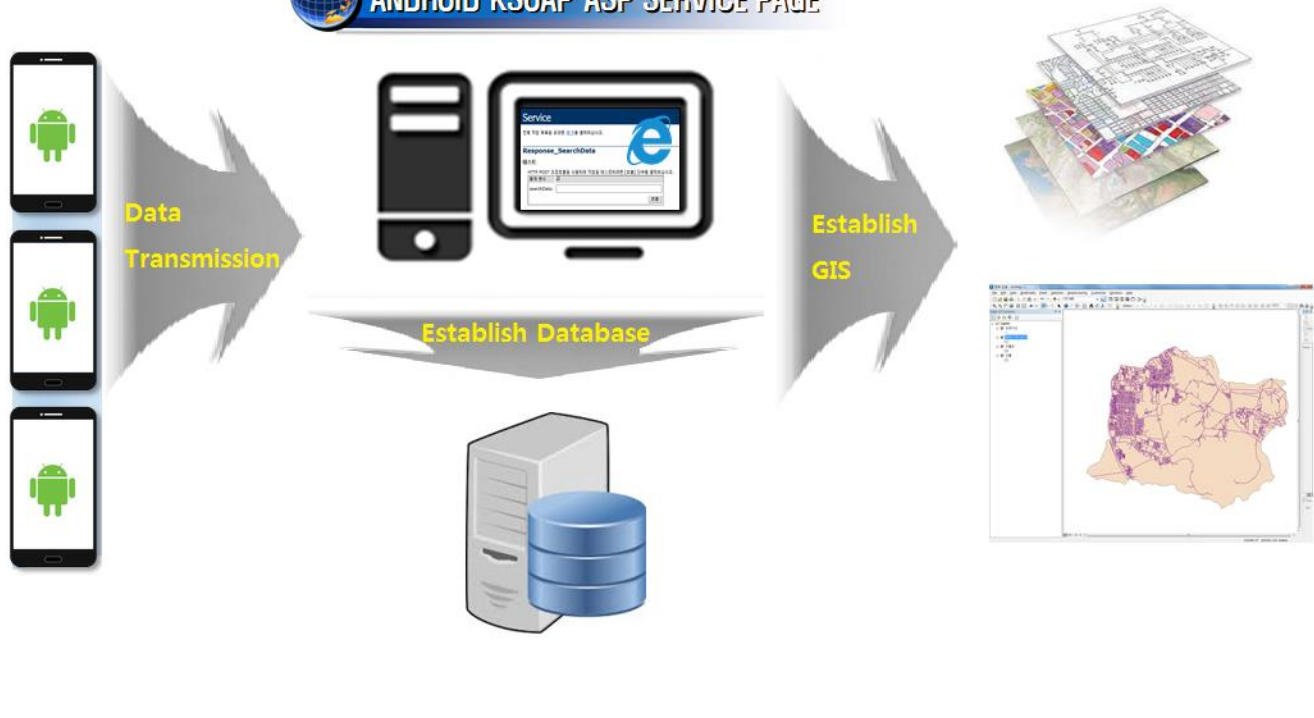

Figure 2. Conceptual Biagram of Transmission and Management System of Real-Time Parking Survey Data

Data transmission items were developed to enable the transmission of data being collected in real time for onsite parking surveys through smartphone communication. This make it possible for several parking inspectors to collect data at the same time from their respective survey positions and transmit this data concurrently in real time. The system was developed so that parking inspectors' data could be transmitted to the Android KSOAP ASP page, and changed to a Query form in the Android KSOAP ASP page to enable the final delivery to the database.

\subsection{Data Analysis and Display System}

In this study, a method for the real time data collection of actual parking conditions, and the analysis and display of stored data was developed to enable a variety of displays using the application program, where it can use coordinate information, such as Arc GIS and Auto CAD, by calling up the query delivered to the database. Figure 3 gives an example of a data table, which is displayed in Arc GIS of parking survey data. Referring to Figure 3, the management of survey data occurs in real time because it shows the inspector's name, block number, street number, survey time, vehicle license number, vehicles type, legal/illegal parking status, and latitude and longitude. 


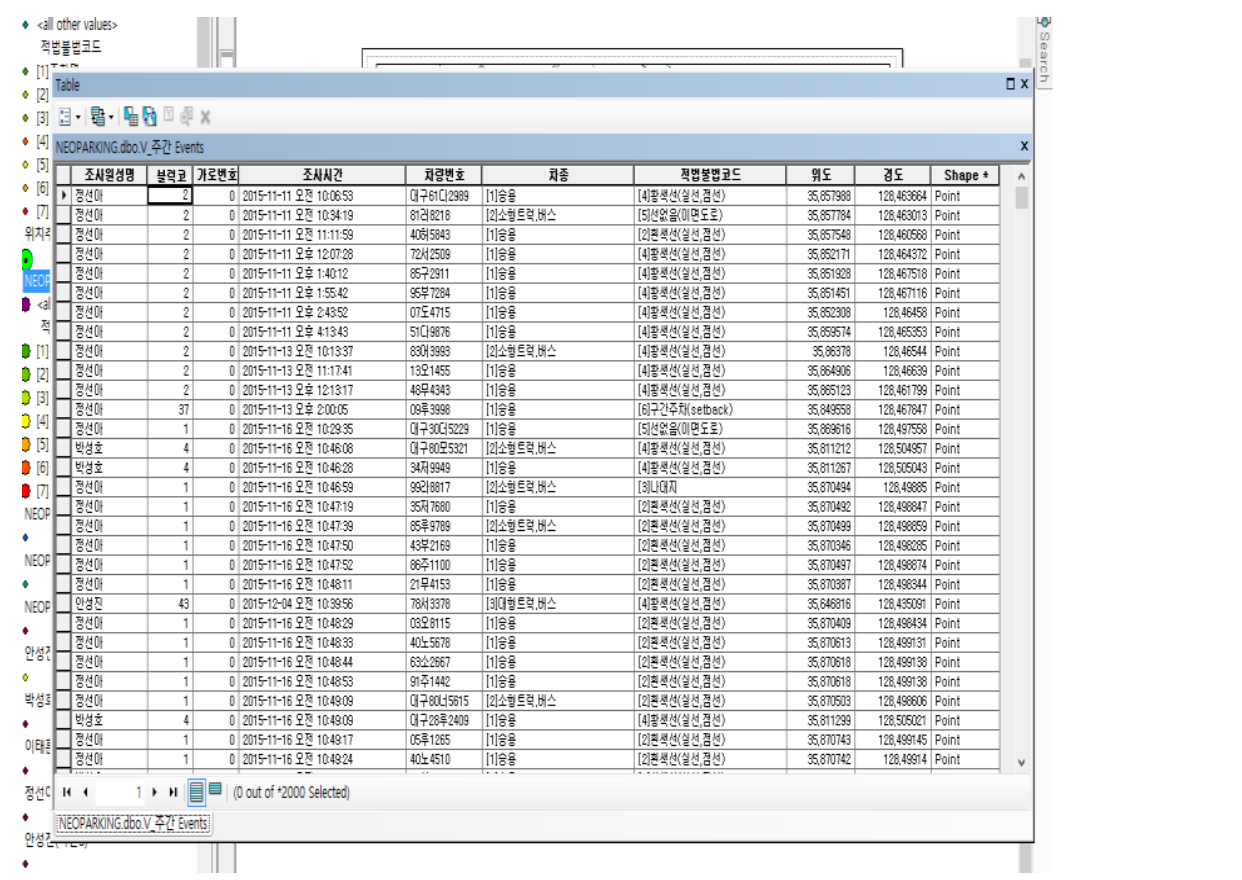

\section{Figure 3. Data Table of Parking Survey Results using the Applied Program}

Using the parking survey data displayed in the data table format, it is possible show the results in dotted form on a map. This will be useful in a variety of applications, such as fact finding parking surveys and analysis because it 18 possible to color-code different vehicle types, and legal/illegal parking satus. In addition, it is possible to visually express the results of the analysis, so it is expected to be used effectively to spatially analyze and understand the parking situation Fgure 4 prevides an example of the analysis of parking survey data and Figure 5 illustrates it on a map.

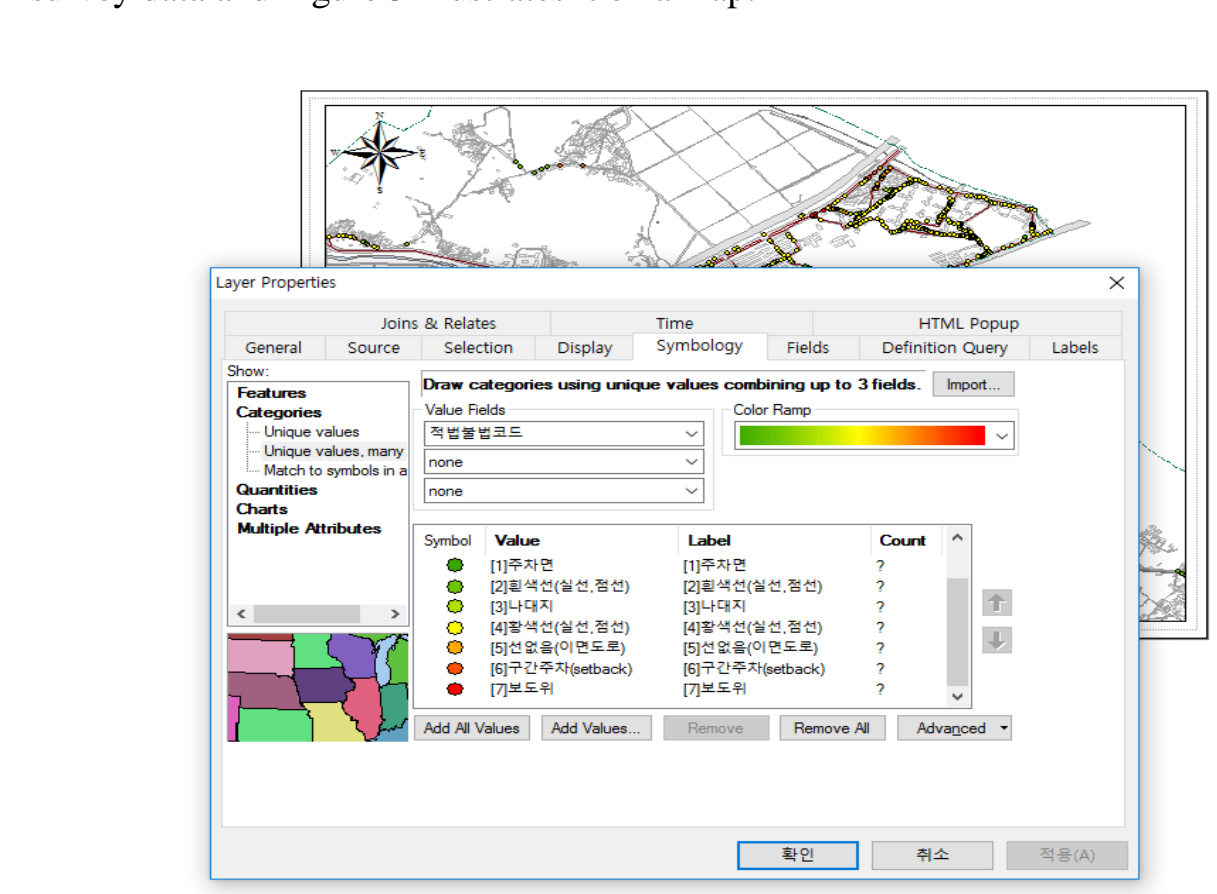

Figure 4. Analysis of the Parking Survey Data 




Existing parking surveys that require inspectors to record their data on paper require too much time and money and have problems such as decreased accuracy and reliability. Therefore, this study arried out resêarch on a parking survey method using a smartphone to overcome the limitations and solve the problems of existing parking surveys. Through the development of a parking survey smartphone application, a system was constructed to allow the easy input of the results of onsite surveys, and the transmission of this real-time data to the server through wireless data transmission.

The survey items of the developed smartphone application for parking surveys provides information on the inspector, survey area, identification of parked vehicles, and location, and was subdivided into the inspector's name, block number, street number, vehicle type, legal/illegal parking status, vehicle license number, vehicle type, and the vehicle's lattude and longitude. The system was developed as a method that enabled vehicles to be input as one of three types, and there were seven different parking statuses poss(ble. To minimize the cost and time for data input and analysis, a system was built to transfer the data collected on -site in real time to the server by wireless transmission. The system was constructed to allow multiple inspectors to send their collected data simultaneously.

The data transmitted in real time is organized automatically into tables and was implemented so that by color-coding in accordance with whether the vehicle was parked legally or illegally, it could be displayed on a map.

This study was carried out to address the problems with the existing time consuming and expensive parking survey methods. If the results of this study can be applied to various parking surveys, such as a survey of the supply and demand for parking lots, which is legally required by cities or various development projects, then it is expected to 
be effective in reducing the social costs through more efficient parking surveys that have been increased in demand.

In the case using the developed real time parking survey data transmission and analysis system, which is the result of this research, it is expected that the proposed system will prevent unnecessary duplicated surveys by the efficient management of collected parking survey data. Furthermore, through the tabulation and visualization of data through maps, it is expected to help understand more comprehensively and three-dimensionally the actual parking situation in the targeted survey area. In addition, it will contribute to establishing a more effective parking policy.

\section{Acknowledgments}

This research was supported by the Daegu University Research Grant, 2016.

\section{References}

[1] H. C. Lee, S. Y. Shin and K. S. Shin, "A Study on Understanding Parking Turnover throug Parking Survey", Journal of the Korea Institute of Information and Communication Engineering, Korea Institute of Information and Communication Engineering, vol. 18, no. 11 (2014), pp. 26452650.

[2] I. C. Hwang, I. H. Kang, and S. G. Lim, "The Effect of Illegal Parking on Residenial Area Roads and Arterial Roads on Traffic", Journal of the Korean Society of C1vil Engineer Korean Society of Civil Engineers, vol. 30, no. 5, (2010), pp. 485-496.

[3] K. H. Lee, S. Y. Shin and B. S. Choi, "Efficient Parking Management through the Investigation of Car License Plate Using Camera", Journal of the Korea Soclety of Computer and Information, Korea Society of Computer and Information, vol. 18, no.11, (2013), pp. 145-151.

[4] K. H. Park, W. H. Hong and J. M. Ha, "A Studyon the Parking Demand Analysis according to Housing Size and Locations in Multi-Family Housing Estates", Journal of Architectural Institute of Korea. Architectural Institute of Korea, vol. 15, no.10, (1999), pp. 21-130.

[5] S. D. Kwon, D. B. Ko, J. J. Park and T J.Ha, "Development of Estimation Models for Parking UnitsFocused on Gwangju Metropolitan City Condominium Apartments", Journal of the Korean Society of Civil Engineers. Korean Society of Civil Engineers, vol. 34, no. 2, (2014), pp. 549-559.

[6] W. Y. Ahn and S. H. Lee, A Study on industrial site annexed parking unit calculation method by considering facility use and scale characte istics;, Journal of Korean Society of Road Engineering. Korean Society of Road ngineering, vol 12, no. 2, (2010), pp. 129-136.

[7] Y. W. Lee, "Calculation Methods of Parking Demand for Housing using Parking Basic Units Analysis", Journal of the Korean Society of Transportation. Korean Society of Transportation, vol. 26, no. 4, (2008), pp. 149-157

[8] Y. W. Lee, "Establishment of an On-street Parking Demand Estimation Model through a Survey on Parking during Day and Night", The Asian International Journal of Life Sciences. Asia Life Science, vol. 12, (2015), pp. 471-483.

[9] Y. W. Lee, "Study on Establishment of On-street Parking Demand Model using Data by Automatic Parking Survey System, International Journal of Applied Engineering Research. Research India Publications, vol. 19. Do. 24, (2014), pp. 24921-24931.

[10] Y. W. Lee, "Study on the Variables for On-street Parking Demand Estimation through Parking Survey", Proceedings of the International Workshop of Architecture and Civil Engineering 2015, Jeju Island, Republic of Korea, (2015) August 43-46.

[11] Y. W. Wee, "Study on Setup Parking Survey Item for Smartphone Application Development", Asiapacific Proceedings of Applied Science and Engineering for Better Human Life, Jeju Island, Republic of Korea, (2016) August 87-90.

12 Y. W. Lee, "Study on Software Development Analyzing the Parking Data Based on an Open API Map", Information-An International Interdisciplinary Journal. International Information Institute, vol. 18, no. 6(B), (2015), pp. 2831-2836.

[13] Y. W. Lee and H. J. Kwon, "A Study on Development and Utilization of Automatic Parking Survey System", Journal of the Korean Society of Transportation. Korean Society of Transportation, vol. 32, no. 5, (2014), pp. 452-461. 


\section{Author}

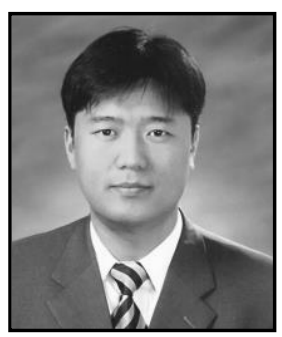

Youngwoo Lee, Associate Professor at Daegu University

lyw209@daegu.ac.kr




International Journal of Smart Home

Vol.10, No.10 (2016)

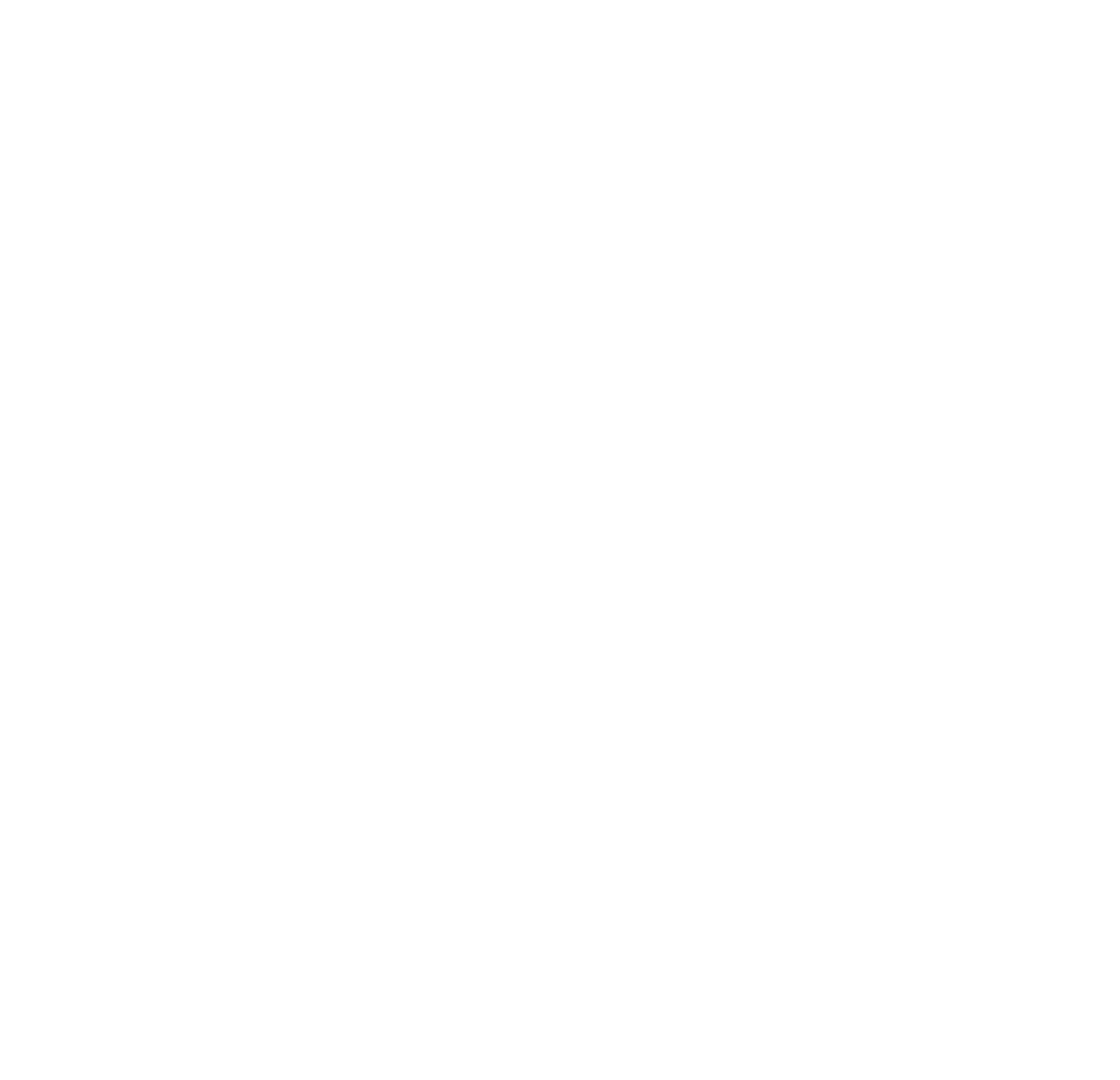

